\title{
Fully-Automated Quantification of Regional Brain Volumes for Improved Detection of Focal Atrophy in Alzheimer Disease
}

\author{
J.B. Brewer \\ S. Magda \\ C. Airriess \\ M.E. Smith
}

\begin{abstract}
SUMMARY: Volumetric analysis of structural MR images of the brain may provide quantitative evidence of neurodegeneration and help identify patients at risk for rapid clinical deterioration. This note describes tests of a fully automated MR imaging postprocessing system for volumetric analysis of structures (such as the hippocampus) known to be affected in early Alzheimer disease (AD). The system yielded results that correlated highly with independent computer-aided manual segmentation and were sensitive to the anatomic atrophy characteristic of mild AD.
\end{abstract}

I tis well established that Alzheimer disease (AD) and related neurodegenerative disorders are associated with atrophy in the brain that can be reliably detected with quantitative analysis of MR images. Consistent with histologic findings, ${ }^{1}$ quantification of structural MR images can reveal significant atrophy in medial temporal lobe structures. ${ }^{2}$ Such loss of tissue is typically accompanied by increases in ventricular volume, particularly in the inferior portion of the lateral ventricle. Quantitative MR imaging may also aid in the detection of AD-related neuropathologic disorders at the prodromal stage. ${ }^{3}$ Patients with moderately poor performance on 1 or more standardized tests of cognitive function are often classified as having mild cognitive impairment (MCI). ${ }^{4}$ When memory is one of the cognitive domains involved, individuals are at increased risk of converting to AD. Medial temporal atrophy has been found to be predictive of whether a patient with MCI will show rapid clinical decline or remain stable. ${ }^{5,6}$

Such findings imply a need for improved tools for MR imaging volumetric analysis that are suitable for routine clinical use. Furthermore, although many efforts are underway to develop treatments that could prevent $\mathrm{AD}$ or slow its progression, these efforts are also hindered in part by the lack of practical yet sensitive in vivo biomarkers that may help to demonstrate disease-modifying effects of potential treatments ${ }^{7}$ and to identify those patients most likely to benefit from aggressive intervention. Both of these needs might be better addressed by efficient methods for the quantitative measurement of atrophy in the living brain. This technical note describes a practical, fully automated method to derive regional brain volumes. The system's validity with respect to results obtained with conventional manual segmentation is

Received July 10, 2008; accepted after revision October 12

From the Departments of Radiology and Neurosciences (J.B.B.), University of California, San Diego, San Diego, Calif; and CorTechs Labs (S.M., C.A., M.E.S.), La Jolla, Calif.

This study was supported by grants to M.E.S. from the US National Institute on Aging and the US National Institute on Neurological Disorders and Stroke.

Some results of this study previously presented at: International Conference on Alzheimer's Disease, July 26-31, 2008, Chicago, III.

Please address correspondence to James B. Brewer, MD, PhD, Human Memory Laboratory, University of California, San Diego, 9500 Gilman Dr, MC 0949, La Jolla, CA 92093; e-mail: jbrewer@ucsd.edu

Indicates open access to non-subscribers at www.ajnr.org examined, as is its sensitivity to the neurodegenerative characteristic of AD.

\section{Technique}

No human subjects were recruited specifically for this study; data included MR images from a group of elderly subjects $(n=40)$ randomly selected from the Open Access Series of Imaging Studies ${ }^{8}$ but balanced for sex, age, and diagnosis. Half had been diagnosed with mild probable AD (Clinical Dementia Rating [CDR] scale, 0.5 or 1), and half were healthy control $(\mathrm{HC})$ subjects $(\mathrm{CDR}, 0)$. The average age was 77 years in each group.

For each subject, a set of T1-weighted magnetization-prepared rapid acquisition of gradient echo sequence anatomic images was available that was obtained with use of a $1.5 \mathrm{~T}$ Vision system (Siemens, Erlangen, Germany). These images were processed by the NeuroQuant software package (CorTechs Labs, La Jolla, Calif), a tool that has received US Food and Drug Administration (FDA) 510K clearance for clinical use to measure volumes of brain structures in MR images. The algorithm used by this software package includes 1) a quality-checking step to determine that the MR imaging sequence conforms to the specifications required to perform automated segmentations, 2) correction for gradient nonlinearity ${ }^{9}$ and B1 field inhomogeneity, ${ }^{10}$ and 3) skull stripping followed by automated segmentation of anatomic structures of interest. An active contour, or "snake," model is used for skull stripping. Discrete cosine transform nonlinear registration aligns volumes to a probabilistic atlas customized for labeling anatomy in elderly subjects. Output of the software includes numeric volumes and images that have been annotated with graphic color overlays, with each color representing a specific segmental structure (Fig 1).

The automated segmentation methods used by the software build on widely used semiautomated methods ${ }^{11,12}$ and rely on probabilistic atlas-based methods to provide volumetric analysis of segmental structures (including those listed in the Table). The segmentation procedure assigns a neuroanatomic label to each voxel on the basis of probabilistic information automatically estimated from an atlas. The labeling of each point in space is achieved by finding the segmentation for each dataset that maximizes the probability of input given the previous probabilities from the atlas. The probability of a class at each point is iteratively computed as the probability that the given class appeared at that location in a training set, modulated by the probability of the surrounding configuration of labels in the 6 cardinal directions, times the likelihood of obtaining the subject-specific measured image intensity value from that class. The NeuroQuant processing procedure takes approximately 8 minutes to complete on a con- 


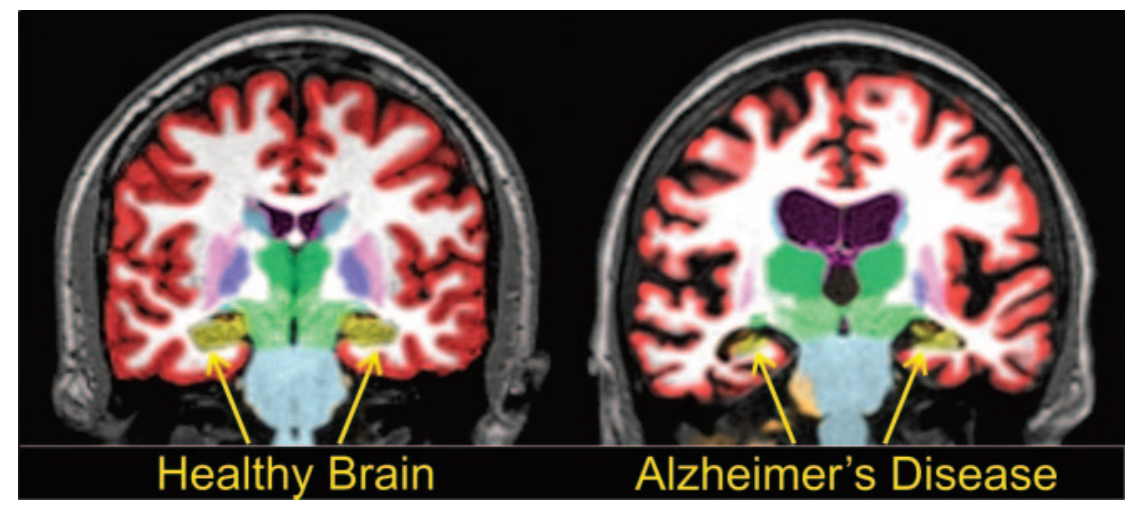

Fig 1. Example coronal sections from automatically segmented MR images from a healthy brain (left) and a patient with AD (right). Arrows indicate medial temporal lobe structures. Colors indicate segmented structures as listed in the Table.

\begin{tabular}{lcc}
\hline \multicolumn{2}{l}{ Manual vs automated segmentation } & \\
\hline Brain Region & ICC & Pearson $r$ \\
\hline Cerebral white matter & $0.98^{*}$ & $0.98^{*}$ \\
Cerebral gray matter & $0.92^{*}$ & $0.93^{*}$ \\
Cerebellum & $0.96^{*}$ & $0.93 \dagger$ \\
Third ventricle & $0.93^{*}$ & $0.92^{*}$ \\
Fourth ventricle & $0.93^{*}$ & $0.90^{*}$ \\
Lateral ventricle & $0.99^{*}$ & $0.99^{*}$ \\
Inferior lateral ventricle & $0.92^{*}$ & $0.89^{*}$ \\
Hippocampus & $0.93^{*}$ & $0.88^{*}$ \\
Amygdala & $0.77^{*}$ & $0.77^{*}$ \\
Thalamus & $0.9^{*}$ & $0.87^{*}$ \\
Caudate & $0.82^{*}$ & $0.75^{*}$ \\
Putamen & $0.89^{*}$ & $0.85^{*}$ \\
Pallidum & $0.76^{*}$ & $0.69^{*}$ \\
Ventral diencephalon & $0.86^{*}$ & $0.80^{*}$ \\
Nucleus accumbens & $0.61 \dagger$ & $0.69^{*}$ \\
Brain stem & $0.93^{*}$ & $0.94^{*}$ \\
\hline
\end{tabular}

Note:-ICC indicates intraclass correlation coefficient.

${ }^{*} P<.001$.

$\dagger P<.002$.

ventional desktop computer and does not require any user input, aside from selecting the study to be segmented. The software interacts directly with a PACS server or can be configured as a remotely hosted server accessed via a Web interface. A report is generated, which returns volumes of structures in cubic centimeters and percentage of intracranial volume. For the hippocampus, lateral ventricle, and temporal horn of the lateral ventricle, a normative range based on previous segmentations of healthy subjects aged 50 to 95 years is provided for reference purposes. An example of full-volume segmentation and 2 example reports are provided as supplementary material.

For comparison purposes, segmentation was also performed independently by a third-party commercial entity (Neuromorphometrics, Somerville, Mass) that was blinded to the diagnosis of patients and to the results of automated segmentation. This entity was paid for its services but had no financial interest in the outcome of the comparison. This segmentation process was conducted with computerassisted semimanual methods by using the open source software package NVM (http://neuromorphometrics.org), which includes features that let the user efficiently delineate and label anatomy in 3 dimensions. Contour drawing and editing were performed by an expert neuroanatomist who used structure boundary definitions as described by the Center for Morphometric Analysis (http://www.cma. mgh.harvard.edu/).

We completed statistical comparisons by using SPSS for Win- dows, Rel. 14. 2005 (SPSS, Chicago, Ill). To evaluate the accuracy of results produced by the automated process relative to segmentation performed in a computer-assisted manual fashion, we computed the intraclass correlation coefficient (ICC) by using Cronbach alpha for each segmented structure obtained (Table 1). ICCs ranged from a low of $r=0.61$ for the nucleus accumbens region $(1$-tailed $P<.002)$ to $r=0.99$ for the lateral ventricle $(1$-tailed $P<.001)$. A high level of agreement between methods was observed in medial temporal regions that are known to atrophy in the early stages of $\mathrm{AD}$ (inferior lateral ventricle ICC, 0.92 ; hippocampal ICC, $0.93 ; P<.001$ for both). A very similar pattern of agreement between methods was observed with the Pearson product-moment correlation coefficient.

To evaluate sensitivity of the automated method to the neuropathologic characteristic of $\mathrm{AD}$, we performed comparisons between volumetric measures in the $\mathrm{AD}$ and $\mathrm{HC}$ subsets of the overall sample, after correcting for differences in total intracranial volume (ICV). In particular, for each subject the volume of each labeled structure was converted to a percentage of total ICV by dividing it by the sum of the volumes for all labeled structures. Highly significant differences between the 2 groups were observed in medial temporal lobe regions that would be expected to be affected in early stage $A D$, including expansion of the inferior lateral ventricle $(\mathrm{AD}>\mathrm{HC}, \mathrm{t}(38)=3.2 ; \mathrm{P}<$ $.003)$, and atrophy of the hippocampus $(\mathrm{AD}<\mathrm{HC}, \mathrm{t}(38)=-4.7 ; P<$ $.001)$ and amygdala $(\mathrm{AD}<\mathrm{HC}, \mathrm{t}(38)=-4.4 ; P<.001)$. In contrast, no significant differences in volume were observed in structures that would not be expected to be affected in early $\mathrm{AD}$ such as the thalamus $(\mathrm{t}(38)=0.64 ; P=.53$, n.s. $)$ or cerebellum $(\mathrm{t}(38)=0.55 ; P=.58$, n.s. $)$.

\section{Discussion}

Several studies have shown that neurodegenerative disorders are reliably associated with patterns of progressive neural atrophy that can be identified with quantitative analysis of MR images. Volumetric analysis of regional brain structures may thus help characterize a patient's condition by providing objective quantitative evidence of a disease process. Such measures may also have prognostic use, helping to identify patients with the greatest risk for rapid clinical deterioration. However, existing methods to derive quantitative volumetric information from MR images have not been suitable for use outside of small-scale research studies.

The results described above indicate that a practical FDAcleared medical device postacquisition MR imaging analysis system for whole-brain anatomic segmentation and for quantification of the volumes of brain structures known to be af- 
fected in $\mathrm{AD}$ yielded volumetric results that correlated highly with results obtained by independent, expert computer-aided manual anatomic segmentation, and were sensitive to differences in volume between age-matched healthy control subjects and patients with early AD. Normative data from younger subjects will help extend these results to other diseases, such as traumatic brain injury, in which measurement of focal brain atrophy may help predict clinical outcome. ${ }^{13}$ However, it should be noted that the presence of large hemorrhage or other gross pathologic processes would likely interfere with automated segmentation of adjacent regions. Nevertheless, the results suggest that a fully automated system accurately measures brain segmental volumes in patients with $\mathrm{AD}$, and this procedure can be applied to T1-weighted volumes from common clinical platforms regardless of field strength. Technology for volumetric MR imaging measurements may now be sufficiently mature to incorporate objective metrics of brain atrophy into everyday clinical practice.

\section{References}

1. Braak H, Braak E. Neuropathological stageing of Alzheimer-related changes. Acta Neuropathol 1991;82:239-59

2. Jack CR Jr, Petersen RC, Xu YC, et al. Medial temporal atrophy on MRI in normal aging and very mild Alzheimer's disease. Neurology 1997;49:786-94
3. Hampel H, Burger K, Teipel SJ, et al. Core candidate neurochemical and imaging biomarkers of Alzheimer's disease. Alzheimers Dement 2008;4:38-48

4. Petersen RC. Mild cognitive impairment as a diagnostic entity. J Intern Med 2004;256:183-94

5. Jack CR Jr, Shiung MM, Weigand SD, et al. Brain atrophy rates predict subsequent clinical conversion in normal elderly and amnestic MCI. Neurology 2005;65:1227-31

6. Devanand DP, Pradhaban G, Liu X, et al. Hippocampal and entorhinal atrophy in mild cognitive impairment: prediction of Alzheimer disease. Neurology 2007;68:828-36

7. Salloway S, Mintzer J, Weiner MF, et al. Disease-modifying therapies in Alzheimer's disease. Alzheimers Dement 2008;4:65-79

8. Marcus DS, Wang TH, Parker J, et al. Open Access Series of Imaging Studies (OASIS): cross-sectional MRI data in young, middle aged, nondemented, and demented older adults. J Cogn Neurosci 2007;19:1498-507

9. Jovicich J, Czanner S, Greve D, et al. Reliability in multi-site structural MRI studies: effects of gradient non-linearity correction on phantom and human data. Neuroimage 2006;30:436-43

10. Sled JG, Zijdenbos AP, Evans AC. A nonparametric method for automatic correction of intensity nonuniformity in MRI data. IEEE Trans Med Imaging 1998; 17:87-97

11. Dale AM, Liu AK, Fischl BR, et al. Dynamic statistical parametric mapping: combining fMRI and MEG for high-resolution imaging of cortical activity. Neuron 2000;26:55-67

12. Fischl BR, Salat DH, Busa E, et al. Whole brain segmentation: automated labeling of neuroanatomical structures in the human brain. Neuron 2002;33:341-55

13. Adamson DM, Burnam MA, Burns RM, et al. Invisible Wounds of War: Psychological and Cognitive Injuries, Their Consequences, and Services to Assist Recovery. Santa Monica: RAND Corp.; 2008 